\title{
ErbB Family Pathway Deregulation
}

National Cancer Institute

\section{Source}

National Cancer Institute. ErbB Family Pathway Deregulation. NCI Thesaurus. Code C138058.

Aberrant activation of an ERBB family member-dependent signaling pathway. 